\title{
Incidence and significance of psammoma bodies in Xp11.2 translocation renal cell carcinoma and papillary renal cell carcinoma
}

\author{
NING LIU $^{1 *}$, FENG QU $^{1 *}$, KANG WEI $^{1}$, WEIDONG GAN ${ }^{1}$, ZHEN WANG $^{1}$, \\ WENYUAN ZHUANG $^{1}$, SEZIM AGIZAMHAN ${ }^{1}$, WENLIANG MA ${ }^{1}$, JUN YANG $^{2}$, \\ MING CHEN $^{2}$, LINFENG XU ${ }^{1}$, HONGQIAN GUO ${ }^{1}$ and DONGMEI LI ${ }^{3,4^{*}}$

\begin{abstract}
Departments of ${ }^{1}$ Urology and ${ }^{2}$ Pathology, Nanjing Drum Tower Hospital, The Affiliated Hospital of Nanjing University Medical School, Nanjing, Jiangsu 210008; ${ }^{3}$ Immunology and Reproduction Biology Laboratory and State Key Laboratory of Analytical Chemistry for Life Science, Nanjing University Medical School, Nanjing, Jiangsu 210093;

${ }^{4}$ Jiangsu Key Laboratory of Molecular Medicine, Nanjing University, Nanjing, Jiangsu 210000, P.R. China
\end{abstract}

Received March 16, 2018; Accepted April 11, 2019

DOI: $10.3892 / \mathrm{ol} .2019 .10305$

\begin{abstract}
The aim of the present study was to investigate the incidence and significance of psammoma bodies (PBs) in Xp11.2 translocation renal cell carcinoma (Xp11.2 tRCC) and papillary renal cell carcinoma (PRCC). The presence of PBs, irregular calcifications, hyaline globules and nested architecture in RCC tissues, which included 47 cases of Xp11.2 tRCC and 95 cases of PRCC, was examined by two pathologists. Compared with PRCC, patients with Xp11.2 tRCC exhibited a higher frequency of PBs, hyaline globules and nested architecture. The presence of PBs in combination with the occurrence of a nested architecture achieved a specificity of $93.7 \%$ when
\end{abstract}

Correspondence to: Dr Weidong Gan, Department of Urology, Nanjing Drum Tower Hospital, The Affiliated Hospital of Nanjing University Medical School, 321 Zhongshan Road, Nanjing, Jiangsu 210008, P.R. China

E-mail: gwd@nju.edu.cn

Professor Dongmei Li, Immunology and Reproduction Biology Laboratory and State Key Laboratory of Analytical Chemistry for Life Science, Nanjing University Medical School, 22 Hankou Road, Nanjing, Jiangsu 210093, P.R. China

E-mail: lidm@nju.edu.cn

*Contributed equally

Abbreviations: AJCC, American Joint Committee on Cancer; FISH, fluorescence in situ hybridization; OS, overall survival; PBs, psammoma bodies; PRCC, papillary renal cell carcinoma; PSF, progression-free survival; PSM, propensity score matching; PTC, papillary thyroid carcinoma; RCC, renal cell carcinoma; Xp11.2 tRCC, Xp11.2 translocation renal cell carcinoma

Key words: psammoma bodies, hyaline globules, renal cell carcinoma, Xp11.2 translocation, propensity score matching diagnosing Xp11.2 tRCC. However, there were no significant differences in the overall survival between patients with and without PBs in both types of RCC. Therefore, the presence of PBs combined with nested architecture may provide guidance for the diagnosis of Xp11.2 tRCC; however, PBs cannot predict tumor behavior in Xp11.2 tRCC or PRCC.

\section{Introduction}

Psammoma bodies (PBs) are basophilic structures with laminated concretions, with a diameter generally ranging from 20-100 $\mu \mathrm{m}$ (1). PBs are composed of calcium apatite and are most commonly observed in papillary thyroid carcinoma (PTC), meningioma, and papillary serous cystadenocarcinoma of the ovary (2). Studies on serous cystadenocarcinoma of the ovary and meningioma demonstrated that collagen production by neoplastic cells and subsequent calcification led to the formation of PBs $(2,3)$. However, an ultrastructural study of PTC revealed that tumor cell necrosis was responsible for the formation of PBs $(2,4)$. Although various theories have been published to explain the origin of PBs, the underlying biochemical mechanism is poorly understood (2-4).

PBs have been reported in $\sim 50 \%$ of PTC cases, which makes it an important marker for the pathological diagnosis of PTC (5). In addition, PBs are of great significance in the diagnosis of meningiomas (6) and serous cystadenocarcinomas of ovary (2), with incidence rates of 45 and 33\%, respectively. Although PBs from various types of neoplasms share identical light-microscopic characteristics, their significance in diverse types of tumors should be further investigated. Cai et al (7) found PBs to be useful predictors of aggressive tumor behavior and patients with PTC with PBs had a poorer prognosis compared with those without PBs (7). However, Das (2) reported that PBs not only lead to degeneration or death of tumor cells, but serve as a barrier against the spread of the neoplasm.

In renal cell carcinoma (RCC), $\mathrm{PBs}$ are most commonly observed in Xp11.2 translocation RCC (Xp11.2 tRCC) (8) and 
papillary (P)RCC (9). Xp11.2 tRCC, a rare and aggressive type of cancer, harbors a chromosomal translocation involving the Xp11.2 breakpoint and transcription factor binding to IGHM enhancer 3 (TFE3) gene fusion $(10,11)$. Microscopically, Xp11.2 tRCC mimics clear cell and PRCC $(10,11)$. The typical morphology of Xp11.2 tRCC includes PBs, irregular calcifications, hyaline globules and nested architecture, particularly for the subtype with the UBX domain containing tether for SLC2A4 (ASPL)-TFE3 gene fusion $(10,12)$. Furthermore, hyaline globules are considered as precursors of PBs and irregular calcifications in PCT (13). However, to the best of our knowledge, the incidence and significance of PBs, irregular calcifications, hyaline globules and nested architecture in Xp11.2 tRCC have only been described in reports with low patient numbers. Therefore, the aim of the present study was to investigate the association between the presence of these specific morphologies and clinicopathological characteristics. A group of PRCC cases were further enrolled due to the high incidence of PBs (9).

\section{Materials and methods}

Ethics approval. All procedures were approved by the Medical Ethics Committee for Human Experiments of Nanjing Drum Tower Hospital (Nanjing, China).

Diagnostic methods. A total of 47 patients with Xp11.2 tRCC were selected between January 2007 and September 2017 at the Nanjing Drum Tower Hospital. Patients were diagnosed by applying self-designed break-apart TFE3 fluorescence in situ hybridization (FISH) probes. Briefly, ASPL-TFE3 dual-fusion FISH probes were used for diagnosing ASPL-TFE3 tRCC. The break-apart and the dual-fusion FISH probe results were validated by reverse transcription-PCR analysis (13). The inclusion criteria comprised patients with RCC who were positive for TFE3 by immunohistochemistry and FISH. Exclusion criteria comprise patients without integrated clinical and pathological data.

Clinicopathological characteristics. According to the 2016 World Health Organization classification of renal cell carcinoma, patients with PRCC were divided into type 1 (31 cases) and type 2 (64 cases) (14). Of the 95 cases of PRCC, 31 were type 1 and 64 were type 2 . Immediately after excision, tumor samples were fixed in buffered $10 \%$ formalin for at least $24 \mathrm{~h}$, embedded in paraffin, cut into $4-\mu \mathrm{m}$ thick sections and stained by hematoxylin and eosin (0.5-1\%) for 5-20 min at room temperature. The hematoxylin and eosin-stained sections of all 142 cases were retrospectively reviewed by two experienced pathologists. To further investigate the role of PBs in Xp11.2 tRCC and PRCC, each group was divided into two subgroups according to the presence of PBs. Besides, sensitivity, specificity and Youden's index of PB in the diagnosis of Xp11.2 RCC was measured by FISH. Clinical data including patient age at onset, sex, maximum tumor diameter, American Joint Committee on Cancer (AJCC) stage (15) and surgical procedure were obtained from reviewing medical records.

Survival data. All patients were followed every 3 months during the first year, every 6 months during the following
4 years and annually after 5 years until the time of death or the loss of follow-up. The survival data were obtained from latest follow-up information. Progression-free survival (PFS) was defined as the time interval between the date of surgery and the date of disease-progression or censoring at the time of the last follow-up. Overall survival (OS) was defined from the date of surgery to the mortality date or the last follow-up.

Statistical analysis. Continuous variables are presented as mean \pm standard deviation and were compared using Student's t-test or one way analysis of variance followed by Student-Newman-Keuls post-hoc test in case of multiple group comparisons. Categorical variables were expressed as counts and percentages, and $\chi^{2}$ or Fisher exact test were used for comparisons. Due to selection bias between subgroups, propensity score matching (PSM) was preformed to balance the distribution of covariates. A non-parsimonious logistic regression model encompassing patients' age, sex, tumor diameter, AJCC stage, surgical procedure and tumor subtype was used to calculate a propensity score for each patient. A 1:1 matching ratio was used in the propensity analyses. Following PSM, matched covariates were re-compared between the two groups with statistical tests. Subsequently, PFS and OS curves were obtained by Kaplan-Meier analysis and statistic comparisons were undertaken using the log-rank test.

In all tests, a two-sided $\mathrm{P}<0.05$ was considered to indicate statistically significant differences. Statistical analysis was performed with SPSS software version 23.0 (IBM Corp.). GraphPad Prism software version 5.0 (GraphPad Software, Inc.) was used for graphics of survival curves.

\section{Results}

Frequency of PBs in different tumor types. Among the 47 cases of Xp11.2 tRCC, 13 (27.7\%) were identified as ASPL-TFE3 tRCC. The incidence rates of PBs in ASPL-TFE 3 tRCC and type 1 PRCC were 69.2 and $41.9 \%$, respectively. The presence of PBs, irregular calcifications, hyaline globules and nested architecture was determined and recorded (Fig. 1). Table I presents the baseline clinical characteristics and frequency of PBs, irregular calcifications, hyaline globules and nested architecture in all the patients. In the Xp11.2 tRCC group, the incidence rate of PBs, hyaline globules and nested architecture was significantly higher compared with that in PRCCs (all $\mathrm{P}<0.05$ ).

Association of other morphological characteristics with PBs. Hyaline globules were present in 22 cases (88.0\%) of Xp11.2 tRCC with PBs, as opposed to $13(59.1 \%)$ cases without PBs $(\mathrm{P}=0.053)$. In addition, $91.7 \%$ (33/36) cases of Xp11.2 tRCC displayed PBs or irregular calcifications, as opposed to $18.2 \%$ $(2 / 11)$ cases without PBs $(\mathrm{P}<0.001)$. Similarly, a significantly higher number of PRCC cases with PBs or irregular calcifications contained hyaline globules, as opposed to cases without PBs or irregular calcifications [45/54 (83.3\%) vs. 8/41 (19.5\%), respectively; $\mathrm{P}<0.001]$. In addition, the sensitivity, specificity and Youden's index of PBs in diagnosing Xp11.2 tRCC were 53.2, 69.5 and $22.7 \%$ respectively. For nested architecture, another histopathological characteristic of Xp11.2 tRCC, the sensitivity, specificity and Youden's index were 
Table I. Clinicopathological characteristics of Xp11.2 tRCC and PRCC.

\begin{tabular}{|c|c|c|c|}
\hline Characteristic & $\mathrm{Xp} 11.2 \operatorname{tRCC}(\mathrm{n}=47)$ & $\operatorname{PRCC}(\mathrm{n}=95)$ & P-value \\
\hline Age (years) & $31.32 \pm 15.66$ & $54.47 \pm 14.02$ & $<0.001$ \\
\hline Sex & & & 0.050 \\
\hline Male & $20(42.6)$ & $25(26.3)$ & \\
\hline Female & $27(57.4)$ & $70(73.7)$ & \\
\hline Tumor size (cm) & $5.43 \pm 2.39$ & $5.01 \pm 2.60$ & 0.360 \\
\hline AJCC stage & & & 0.608 \\
\hline 1 & $29(61.7)$ & $63(66.3)$ & \\
\hline 2 & $4(8.5)$ & $8(8.4)$ & \\
\hline 3 & $11(23.4)$ & $18(18.9)$ & \\
\hline 4 & $3(6.4)$ & $6(6.3)$ & \\
\hline Psammoma body & $25(53.2)$ & $29(30.5)$ & 0.009 \\
\hline Irregular calcification & $29(61.7)$ & $42(44.2)$ & 0.050 \\
\hline Hyaline globule & $35(74.5)$ & $53(55.8)$ & 0.031 \\
\hline Nested architecture & $16(34.0)$ & $14(14.7)$ & 0.008 \\
\hline
\end{tabular}

Data are presented as $\mathrm{n}(\%)$ or the mean \pm standard deviation. AJCC, American Joint Committee on Cancer; PRCC, papillary renal cell carcinoma; Xp11.2 tRCC, Xp11.2 translocation renal cell carcinoma.

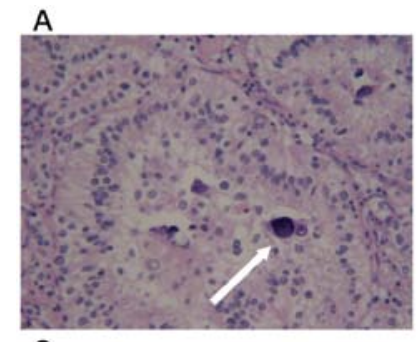

C
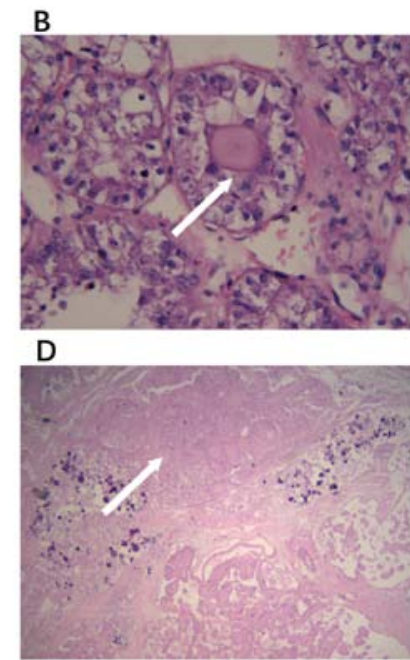

Figure 1. PBs, irregular calcifications, hyaline globules and nested architecture in tissue samples from Xp11.2 translocation renal cell carcinoma. Typical appearance of (A) PB with laminated a structure (white arrow; magnification, $\mathrm{x} 400$ ), (B) hyaline globules surrounded by a layer of neoplastic cells (white arrow; magnification. $\mathrm{x} 400),(\mathrm{C})$ irregular calcifications (highlighted using white arrow; magnification, x100) and (D) irregular calcification deposits outside the nested formations (highlighted using white arrow; magnification, x100). PBs, psammoma bodies.

34.0, 85.3 and $19.3 \%$, respectively. When PBs were combined with nested architecture, the specificity in diagnosing Xp11.2 tRCC increased to $93.7 \%$; however, sensitivity dropped to $17.0 \%$ (Table II).

The clinicopathological characteristics of patients with Xp11.2 tRCC and PRCC before and after PSM are summarized in Tables III and IV, respectively. Prior to PSM, only the AJCC stages in the PRCC group were proven to differ significantly between the groups with and without $\mathrm{PB}(\mathrm{P}=0.015)$. After PSM, 44 patients with Xp11.2 tRCC and 58 with PRCC were selected and no significant differences in clinicopathological characteristics, including patient age, sex distribution, tumor size, AJCC stage and subtype was associated with the presence of PBs.

Follow-up and survival data. Except for two patients, no patients were lost to follow-up. The mean follow-up duration was 44.69 \pm 31.56 months (range 5-121 months) in the Xp11.2 tRCC group and 27.23 \pm 21.40 months (range 1-89 months) in the PRCC group. During follow-up, 14 patients with Xp11.2 tRCC (29.8\%) and 22 patients with PRCC (23.2\%) showed disease progression. The 5-year OS rate for patients with Xp11.2 tRCC and PRCC was 81.8 and $67.1 \%$, respectively $(\mathrm{P}=0.196)$. After adjusting the covariates using PSM, there were no statistical differences in the survival curves for PFS and OS based on PB occurrence (Fig. 2).

\section{Discussion}

Since PBs were first described, various mechanisms underlying PB morphogenesis have been suggested and the hypothesis of dystrophic calcification appears to be the most likely explanation. During the process of dystrophic calcification, events such as thickening of the basal lamina, vascular thrombosis, calcification and tumor cell necrosis occur sequentially (4). The formation of papillae, where the precursors of PBs usually originate, is an important morphological characteristic associated with PBs (2). After analyzing the IgG distribution of blood vessels, whorls and PBs of meningiomas, Tabuchi et al (16) hypothesized that humoral immunity participates in the formation of whorls and PBs. Another important contributor of the formation of PBs is type IV collagen (3). Described by a nested formation around the basement membrane, type IV collagen may be involved in anchoring PBs to the site of development of meningiomas (3). Additionally, type IV collagen was found 
Table II. The role of psammoma bodies and nested architecture in diagnosing Xp11.2 tRCC.

\begin{tabular}{lccc}
\hline Characteristic & Sensitivity (\%) & Specificity (\%) & Youden's index (\%) \\
\hline Psammoma body & 53.2 & 69.5 & 22.7 \\
Nested architecture & 34.0 & 85.3 & 19.3 \\
Psammoma body + Nested architecture & 93.7 & 17.0 & 10.7 \\
\hline
\end{tabular}

Table III. Comparisons of clinicopathological characteristics of Xp11.2 tRCC based on the presence of PB.

\begin{tabular}{|c|c|c|c|c|c|}
\hline \multirow[b]{2}{*}{ Characteristic } & \multirow[b]{2}{*}{ No PB $(n=22)$} & \multicolumn{4}{|c|}{$\mathrm{PB}$} \\
\hline & & All $(n=25)$ & P-value ${ }^{a}$ & PSM-adjusted $(n=22)$ & $\mathrm{P}$-value ${ }^{\mathrm{a}}$ \\
\hline Age $(\text { years })^{b}$ & $29.32 \pm 18.41$ & $32.84 \pm 13.51$ & 0.465 & $32.68 \pm 13.64$ & 0.500 \\
\hline $\operatorname{Sex}^{\mathrm{c}}$ & & & 0.831 & & 1 \\
\hline Female & $13(59.09)$ & $14(56.00)$ & & $13(59.09)$ & \\
\hline Male & $9(40.91)$ & $11(44.00)$ & & $9(40.91)$ & \\
\hline Tumor size $(\mathrm{cm})^{\mathrm{b}}$ & $6.00 \pm 2.55$ & $4.92 \pm 2.17$ & 0.166 & $5.03 \pm 2.28$ & 0.173 \\
\hline $\mathrm{AJCC}_{\text {stage }} \mathrm{c}^{\mathrm{c}}$ & & & 0.458 & & 0.666 \\
\hline 1 & $12(54.55)$ & $17(86.00)$ & & $14(63.64)$ & \\
\hline 2 & $3(13.64)$ & $1(4.00)$ & & $1(4.55)$ & \\
\hline 3 & $5(22.72)$ & $6(24.00)$ & & $6(27.26)$ & \\
\hline 4 & $2(9.09)$ & $1(4.00)$ & & $1(4.55)$ & \\
\hline Subtype ${ }^{c}$ & & & 0.173 & & 0.173 \\
\hline$A S P L$ & $4(18.18)$ & $9(36.00)$ & & $8(36.36)$ & \\
\hline Non- $A S P L$ & $18(81.82)$ & $16(64.00)$ & & $14(63.64)$ & \\
\hline
\end{tabular}

Data are presented as $\mathrm{n}(\%)$ or the mean \pm standard deviation. ${ }^{\mathrm{a}} \mathrm{P}$-value vs. no PB determined using ${ }^{\mathrm{b}}$ analysis of variance or ${ }^{\mathrm{c}} \chi^{2}$ test. AJCC, American Joint Committee on Cancer; Xp11.2, Xp11.2 tRCC translocation renal cell carcinoma; ASPL, UBX domain containing tether for SLC2A4; PBs, psammoma bodies; PSM, propensity score matching.
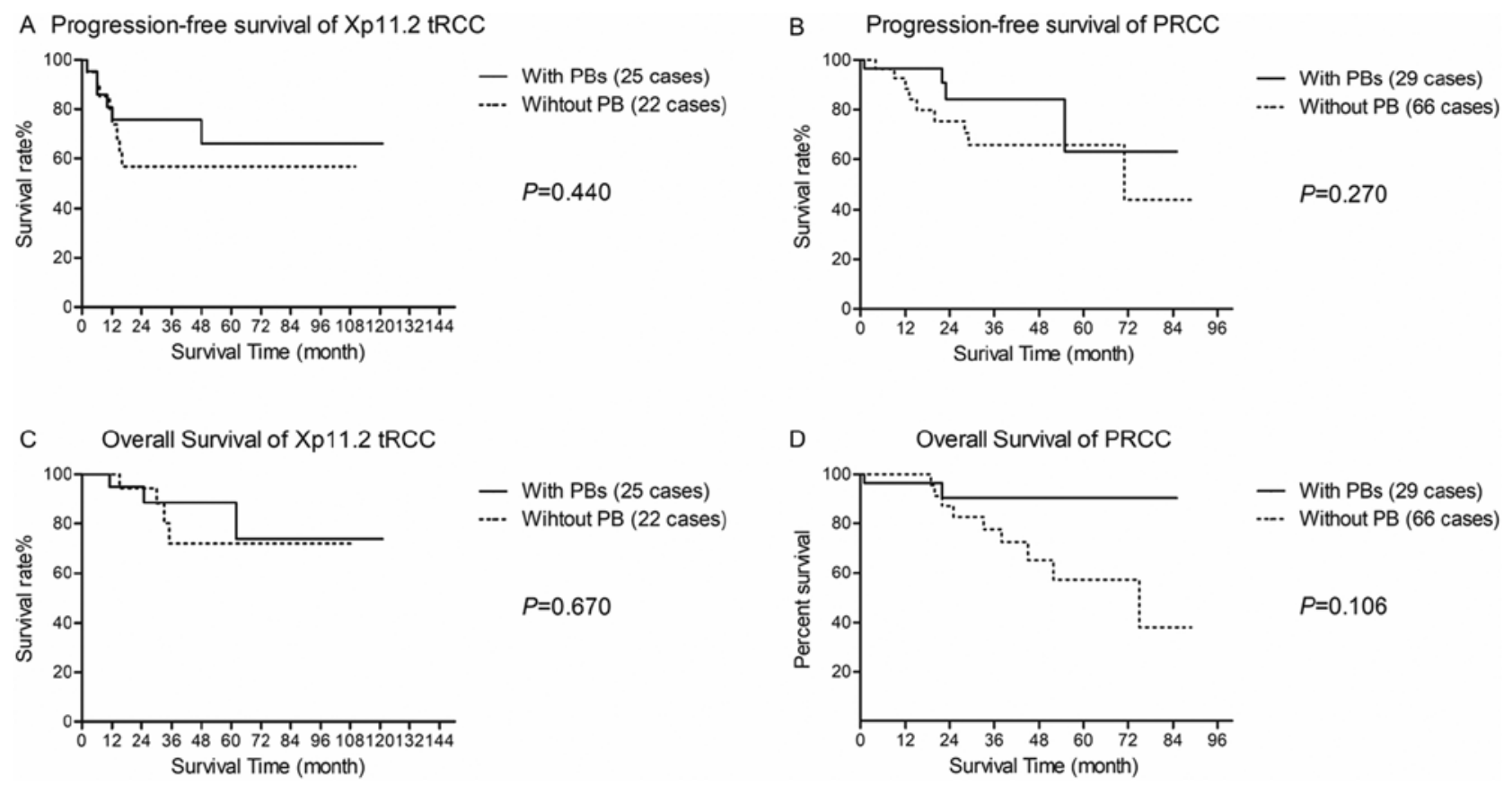

Figure 2. Survival analysis of patients with and without PB. Progression-free survival for patients with (A) Xp11.2 tRCC and (B) PRCC. Overall survival of patients with (C) Xp11.2 tRCC and (D) PRCC. PBs, psammoma bodies; RCC, renal cell carcinoma; PRCC, papillary RCC; Xp11.2 tRCC, Xp11.2 translocation RCC. 
Table IV. Comparisons of clinicopathological characteristics of PRCC based on the presence of PB.

\begin{tabular}{|c|c|c|c|c|c|}
\hline \multirow[b]{2}{*}{ Characteristic } & \multirow[b]{2}{*}{$\mathrm{PB}(\mathrm{n}=29)$} & \multicolumn{4}{|c|}{ No PB } \\
\hline & & Original $(n=66)$ & P-value ${ }^{a}$ & PSM-adjusted $(n=29)$ & P-value \\
\hline Age (years) ${ }^{\mathrm{b}}$ & $52.07 \pm 15.67$ & $55.53 \pm 13.22$ & 0.265 & $59.17 \pm 13.42$ & 0.053 \\
\hline $\operatorname{Sex}^{c}$ & & & 0.409 & & 1 \\
\hline Male & $6(20.69)$ & 19 (28.79) & & $6(20.69)$ & \\
\hline Female & $23(79.31)$ & $47(71.21)$ & & $23(79.31)$ & \\
\hline Tumor size $(\mathrm{cm})^{\mathrm{b}}$ & $4.36 \pm 2.24$ & $5.30 \pm 2.72$ & 0.115 & $5.21 \pm 2.96$ & 0.224 \\
\hline AJCC stage ${ }^{c}$ & & & 0.015 & & 0.058 \\
\hline 1 & $24(82.76)$ & $39(59.09)$ & & $18(62.07)$ & \\
\hline 2 & $3(10.34)$ & $5(7.57)$ & & $4(13.79)$ & \\
\hline 3 & $1(3.45)$ & $17(25.76)$ & & $6(20.69)$ & \\
\hline 4 & $1(3.45)$ & $5(7.57)$ & & $1(3.45)$ & \\
\hline Subtype & & & 0.093 & & 0.097 \\
\hline 1 & $13(44.83)$ & 18 (27.27) & & 7 (24.14) & \\
\hline 2 & $16(55.17)$ & 48 (72.73) & & $22(75.86)$ & \\
\hline
\end{tabular}

Data are presented as $\mathrm{n}(\%)$ or the mean \pm standard deviation. ${ }^{\text {a }} \mathrm{P}$-value vs. PB determined using banalysis of variance or ${ }^{\mathrm{c}} \chi^{2}$ test. PBs, psammoma bodies; AJCC, American Joint Committee on Cancer; PRCC, papillary renal cell carcinoma; PSM, propensity score matching.

Table V. Literature described cases of Xp11.2 tRCC involving psammoma bodies.

\begin{tabular}{lcccc}
\hline Author, year & Patients (n) & Diagnosis methods & Incident rate of PB (\%) & (Refs.) \\
\hline Qu et al, 2016 & 30 & IHC+FISH & 26.7 & $(20)$ \\
Rao et al, 2011 & 19 & IHC+CK & 94.7 & $(21)$ \\
Zou et al, 2014 & 9 & IHC & 77.8 & $(22)$ \\
He et al, 2014 & 6 & IHC & 83.3 & $(23)$ \\
Komai et al, 2009 & 7 & IHC+CK & 42.9 & $(24)$ \\
Argani et al, 2007 & 28 & IHC+PCR & 50.0 & $(10)$ \\
Camparo et al, 2008 & 31 & IHC+CK & 62.0 & $(25)$
\end{tabular}

CK, cytogenetic karyotype; FISH, fluorescence in situ hybridization; IHC, immunohistochemical; Xp11.2 tRCC, Xp11.2 translocation renal cell carcinoma.

to be selectively expressed in PB-forming ovarian cancer cell lines, which suggested that type IV collagen slows down the formation of PBs during the growth of ovarian cancer cells (17).

In RCC, PBs have mainly been reported for Xp11.2 tRCC and PRCC $(8,9)$, and occasionally for chromophobe RCC (18) and renal oncocytoma (19). Unlike the aforementioned studies, which were largely supported by immunohistochemistry and molecular techniques, the present study was a morphological study to summarize the incidence of PBs in PRCC and Xp11.2 tRCC. Published reports of Xp11.2 tRCC with identified PBs are summarized in Table $\mathrm{V}$, to reflect that the incidence rate of $\mathrm{PB}$ in Xp11.2 tRCC varies greatly among different reports (10,20-25). To the best of our knowledge, there has been no previous report about the clinical significance of PBs in Xp11.2 tRCC.

Compared with PRCC, Xp11.2 tRCC shares a similar histopathological structure, but exhibits a more aggressive course $(10,11)$. In the diagnosis of Xp11.2 tRCC, TFE3 immunohistochemical staining serves as the basic method, with high false-positive rates and low predictive values (26). Therefore, FISH, PCR or karyotype analysis is applied for a definitive diagnosis (12). Although frequently present in Xp11.2 tRCC and PRCC, the diagnostic and predictive role of PBs has not yet been fully elucidated $(8,9)$. The present study included 47 cases of Xp11.2 tRCC and 95 cases of PRCC, and demonstrated that the incidence of PBs in Xp11.2 tRCC was significantly higher compared with that in PRCC. The specificity of PBs combined with nested architecture in diagnosing Xp11.2 tRCC reached $93.7 \%$, suggesting a potential diagnostic role in Xp11.2 tRCC screening.

Numerous studies have reported the association between hyaline globules and PBs/irregular calcifications $(3,13,27)$. In a PTC case diagnosed by fine-needle aspiration, 
Haji et al (27) found only few PBs, but numerous laminated hyaline globules. Immunohistochemical staining using paraffin-embedded sections revealed that these globules were positive for von Kossa staining, but negative for thyroglobulin, suggesting an early or precursor form of PBs. In the study from Das et al (13), the presence of hyaline degeneration among cases with and without PBs/irregular calcifications was $80.0 \%$ vs. $22.7 \%$, respectively. Similarly, the statistical analysis of the present study favored the hypothesis that hyaline globules may be precursors of $\mathrm{PBs}$ in Xp11.2 tRCC and PRCC.

The predictive role of PBs has been associated with tumor types (2,7). In the PRCC group, type 1 tumors contained more PBs and displayed a more favorable behavior (9). In the Xp11.2 tRCC group, ASPL-TFE3 tRCC exhibited a higher incidence of PBs compared with other fusion types. However, ASPL-TFE3 tRCC has been reported to be more likely associated with lymph node metastasis and disease progression $(10,11,28)$. Based on this, it is crucial to investigate whether PBs exert opposite effects in different tumor types. However, the PSM data obtained in the present study suggested that the presence of PBs was not associated with any of the observed clinicopathological characteristics, including tumor size, AJCC stage or survival, in PRCC and Xp11.2 tRCC.

To the best of our knowledge, the present study was the first to systematically investigate the incidence and significance of PBs, irregular calcifications, hyaline globules and nested architecture in Xp11.2 tRCC and PRCC. However, there were certain limitations. This was a retrospective study and the sample size of Xp11.2 tRCC was low due to its scarcity; however, it is believed to be one of the largest single-center clinical reports on Xp11.2 tRCC. Additionally, as patients received various adjuvant therapies following surgery, selection bias persisted even after PSM was performed. Furthermore, other subtypes of RCC, including chromophobe RCC and renal oncocytoma, were not included in the present study due to the small number of cases and their favorable prognosis. Further studies are required to elucidate the biochemical mechanisms underlying the development of PBs in RCC.

The incidence rate of PBs in Xp11.2 tRCC was found to be significantly higher compared with that in PRCC, and the presence of hyaline globules was associated with PBs and irregular calcifications. However, the presence of PBs was not found to be associated with tumor subtype or prognosis in Xp11.2 tRCC or PRCC. When PBs and nested architecture were present simultaneously in RCC, additional methods may be used to investigate the potential of Xp11.2 tRCC occurrence.

\section{Acknowledgements}

The authors would like to thank Professor Xiaogong Li, Professor Gutian Zhang, Dr Linfang Yao and Dr Xiaozhi Zhao (Department of Urology, Nanjing Drum Tower Hospital) for providing patient information.

\section{Funding}

The present study was supported by the National Natural Science Foundation of China (grant no. 81572512).

\section{Availability of data and materials}

The datasets used and/or analyzed during the current study are available from the corresponding author on reasonable request.

\section{Authors' contributions}

WG, DL and HG provided the idea and acquired funding for the studies. NL and FQ made substantial contributions to the conception or design of the work. NL, KW, WZ, ZW, SA and $\mathrm{WM}$ analyzed and interpreted the patient data regarding psammoma bodies in Xp11.2 translocation renal cell carcinoma and papillary renal cell carcinoma. FQ, LX, JY and MC performed the histological examinations of the renal cell carcinomas and were major contributors to the writing of the manuscript. WG, LX and HG were involved in drafting and revising the manuscript. All the authors have read and approved the final manuscript.

\section{Ethics approval and consent to participate}

All procedures were approved by the Medical Ethics Committee for Human Experiments of Nanjing Drum Tower Hospital (Nanjing, China). At time of hospitalization, patients provided written informed consent for the collection and use of their tissues.

\section{Patient consent for publication}

The patients, or parents, guardians or next of kin provided written informed consent for the publication.

\section{Competing interests}

The authors declare that they have no competing interests.

\section{References}

1. Fadare O, Chacho MS and Parkash V: Psammoma bodies in cervicovaginal smears: Significance and practical implications for diagnostic cytopathology. Adv Anat Pathol 11: 250-261, 2004.

2. Das DK: Psammoma body: A product of dystrophic calcification or of a biologically active process that aims at limiting the growth and spread of tumor? Diagn Cytopathol 37: 534-541, 2009.

3. Han J, Daniel JC and Pappas GD: Expression of type VI collagen in psammoma bodies: Immunofluorescence studies on two fresh human meningiomas. Acta Cytol 40: 177-181, 1996.

4. Johannessen JV and Sobrinho-Simoes M: The origin and significance of thyroid psammoma bodies. Lab Invest 43: 287-296, 1980.

5. Pyo JS, Kang G, Kim DH, Park C, Kim JH and Sohn JH: The prognostic relevance of psammoma bodies and ultrasonographic intratumoral calcifications in papillary thyroid carcinoma. World J Surg 37: 2330-2335, 2013.

6. Carneiro SS, Scheithauer BW, Nascimento AG, Hirose T and Davis DH: Solitary fibrous tumor of the meninges: A lesion distinct from fibrous meningioma. A clinicopathologic and immunohistochemical study. Am J Clin Pathol 106: 217-224, 1996.

7. Cai YF, Wang QX, Ni CJ, Guo GL, Li Q, Wang OC, Wu L, Du HY, You J and Zhang XH: The clinical relevance of psammoma body and hashimoto thyroiditis in papillary thyroid carcinoma: A large case-control study. Medicine (Baltimore) 94: e1881, 2015.

8. Armah HB and Parwani AV: Xp11.2 translocation renal cell carcinoma. Arch Pathol Lab Med 134: 124-129, 2010.

9. Delahunt B, Eble JN, McCredie MR, Bethwaite PB, Stewart JH and Bilous AM: Morphologic typing of papillary renal cell carcinoma: Comparison of growth kinetics and patient survival in 66 cases. Hum Pathol 32: 590-595, 2001. 
10. Argani P, Olgac S, Tickoo SK, Goldfischer M, Moch H, Chan DY, Eble JN, Bonsib SM, Jimeno M, Lloreta J, et al: Xp11 translocation renal cell carcinoma in adults: Expanded clinical, pathologic, and genetic spectrum. Am J Surg Pathol 31: 1149-1160, 2007.

11. Chen X, Yang Y, Gan W, Xu L, Ye Q and Guo H: Newly designed break-apart and ASPL-TFE3 dual-fusion FISH assay are useful in diagnosing Xp11.2 translocation renal cell carcinoma and ASPL-TFE3 renal cell carcinoma: A STARD-compliant article. Medicine (Baltimore) 94: e873, 2015.

12. Liu N, Wang Z, Gan W, Xiong L, Miao B, Chen X, Guo H and Li D: Renal cell carcinoma associated with Xp11.2 translocation/TFE3 gene fusions: Clinical features, treatments and prognosis. PLoS One 11: e0166897, 2016.

13. Das DK, Mallik MK, Haji BE, Ahmed MS, Al-Shama'a M, Al-Ayadhy B, George SS, Sathar SA and Junaid TA: Psammoma body and its precursors in papillary thyroid carcinoma: A study by fine-needle aspiration cytology. Diagn Cytopathol 31: 380-386, 2004

14. Moch H, Cubilla AL, Humphrey PA, Reuter VE and Ulbright TM: The 2016 WHO classification of tumours of the urinary system and male genital organs-Part A: Renal, penile, and testicular tumours. Eur Urol 70: 93-105, 2016.

15. Balch CM, Gershenwald JE, Soong SJ, Thompson JF, Atkins MB Byrd DR, Buzaid AC, Cochran AJ, Coit DG, Ding S, et al: Final version of 2009 AJCC melanoma staging and classification. J Clin Oncol 27: 6199-6206, 2009.

16. Tabuchi K, Kawakami Y and Nishimoto A: Immunohistochemical demonstration of IgG in meningioma. Acta Neurochir (Wien) 55: 201-211, 1981.

17. Kiyozuka Y, Nakagawa H, Senzaki H, Uemura Y, Adachi S, Teramoto Y, Matsuyama T, Bessho K and Tsubura A: Bone morphogenetic protein-2 and type IV collagen expression in psammoma body forming ovarian cancer. Anticancer Res 21: 1723-1730, 2001

18. Cohen RJ, Weinstein S, Robertson T, Sellner LN, Dawkins HJ and McNeal JE: Variant chromophobe renal cell carcinoma. Arch Pathol Lab Med 124: 904-906, 2000.

19. Amin MB, Crotty TB, Tickoo SK and Farrow GM: Renal oncocytoma: A reappraisal of morphologic features with clinicopathologic findings in 80 cases. Am J Surg Pathol 21: 1-12, 1997.
20. Qu Y, Gu C, Wang H, Chang K, Yang X, Zhou X, Dai B, Zhu Y, Shi G, Zhang $\mathrm{H}$ and Ye D: Diagnosis of adults Xp11.2 translocation renal cell carcinoma by immunohistochemistry and FISH assays: Clinicopathological data from ethnic Chinese population. Sci Rep 6: 21677, 2016.

21. Rao Q, Chen JY, Wang JD, Ma HH, Zhou HB, Lu ZF and Zhou XJ: Renal cell carcinoma in children and young adults: Clinicopathological, immunohistochemical, and VHL gene analysis of 46 cases with follow-up. Int J Surg Pathol 19: 170-179, 2011.

22. Zou H, Kang X, Pang LJ, Hu W, Zhao J, Qi Y, Hu J, Liu C, Li H, Liang W, et al: Xp11 translocation renal cell carcinoma in adults: A clinicopathological and comparative genomic hybridization study. Int J Clin Exp Pathol 7: 236-245, 2014.

23. He J, Huan Y, Qiao Q, Zhang J and Zhang JS: Renal carcinomas associated with Xp11.2 translocations: Are CT findings suggestive of the diagnosis? Clin Radiol 69: 45-51, 2014.

24. Komai Y, Fujiwara M, Fujii Y, Mukai H, Yonese J, Kawakami S, Yamamoto S, Migita T, Ishikawa Y, Kurata M, et al: Adult Xp11 translocation renal cell carcinoma diagnosed by cytogenetics and immunohistochemistry. Clin Cancer Res 15: 1170-1176, 2009.

25. Camparo P, Vasiliu V, Molinie V, Couturier J, Dykema KJ, Petillo D, Furge KA, Comperat EM, Lae M, Bouvier R, et al: Renal translocation carcinomas: Clinicopathologic, immunohistochemical, and gene expression profiling analysis of 31 cases with a review of the literature. Am J Surg Pathol 32: 656-670, 2008.

26. Klatte T, Streubel B, Wrba F, Remzi M, Krammer B, de Martino M, Waldert M, Marberger M, Susani M and Haitel A: Renal cell carcinoma associated with transcription factor E3 expression and Xp11.2 translocation: Incidence, characteristics, and prognosis. Am J Clin Pathol 137: 761-768, 2012.

27. Haji BE, Ahmed MS, Prasad A, Omar MS and Das DK: Papillary thyroid carcinoma with an adenoid cystic pattern: Report of a case with fine-needle aspiration cytology and immunocytochemistry. Diagn Cytopathol 30: 418-421, 2004.

28. Ellis CL, Eble JN, Subhawong AP, Martignoni G, Zhong M, Ladanyi M, Epstein JI, Netto GJ and Argani P: Clinical heterogeneity of Xp11 translocation renal cell carcinoma: Impact of fusion subtype, age, and stage. Mod Pathol 27: 875-886, 2014. 